\title{
CONFERENCIA MUNDIAL SOBRE EDUCACION DE ADULTOS A DISTANCIA
}

Con ocasión de la celebración del décimo aniversario de la Fundación "Open University" de Inglaterra, se llevó a cabo en la ciudad de Birmingham, durante los días 18 al 24 de Noviembre, una Conferencia Internacional sobre Educación de Adultos a Distancia; con la participación de 250 personas representantes de 52 países del mundo, en los cuales se realizan actividades en este campo.

La metodología de trabajo de la conferencia se realizó a través de grupos organizados previamente, de acuerdo a las preferencias de cada uno de los participantes, y según la combinación de las siguientes características: educación superior a distancia, educación a distancia a otros niveles; países subdesarrollados, países desarrollados, combinación de países desarrollados y subdesarrollados.

Los objetivos de la Conferencia fueron:

—Analizar la naturaleza y características de los sistemas a distancia y sus usuarios.

- Analizar la experiencia de colaboración entre instituciones, países y regiones y estudiar sus posibilidades en el futuro.

Los temas desarrollados fueron los siguientes:

1. Población objeto, metas y medios

— ¿Para quién son los programas de educación de adultos a distancia?

—Cuáles son los principales objetivos sociales, políticos, económicos y culturales?

- ¿Qué recursos (humanos, financieros, materiales) son necesarios para ofrecer educación a distancia para tales propósitos y a tales grupos?

— ¿Qué tipo de mareo institucional es el más apropiado para implementar la educación a distancia?

2. Principios generales y metodología de la educación a distancia

— ¿Cuáles son los problemas educativos/pedagógicos de la educación de adultos a distancia?

—QQué enfoques han sido ensayados hasta el presente?

- ¿Qué éxito han tenido estos intentos?

- Teoría y práctica en el diseño de sistema de aprendizaje.

- Medios para la enseñanza y el aprendizaje y su uso sistemático en la educación de adultos a distancia. 


\section{Experiencias de colaboración institucional, nacional regional e internacional}

—El problema de la transferencia de materiales de aprendizaje.

- Sistemas y métodos.

-Experiencias y personas entre instituciones de aprendizaje a distancia e instituciones convencionales; entre instituciones de aprendizaje a distancia.

— ¿Qué se ha logrado hasta el presente? y ¿Cuál es el potencial futuro de esta colaboración?

\section{Posibilidades de colaboración internacional en el futuro}

- Si se considera conveniente la continuación de este tipo de colaboración, ¿cómo debería definirse y llevarse a cabo en el futuro?

La discusión de estos temas estuvo ilustrada y documentada por la experiencia y la exposición de 80 trabajos cortos, los cuales fueron leídos por los participantes.

Además de la riqueza del intercambio proporcionada por las personas que trabajan en esta actividad, en diversas partes del mundo, los grupos produjeron una serie de consideraciones y recomendaciones que están recogidas en informes publicados provisionalmente, pero que serán presentados en un libro a editarse en los próximos meses. La gran sorpresa para muchos participantes, particularmente para quien escribe esta nota, fue el encontrar que el movimiento de educación a distancia tiene ya una dimensión mundial que comienza a impactar los sistemas tradicionales de enseñanza, los cuales se verán forzados a renovar y redefinir su concepción filosófica y sus métodos educativos.

Sin pretender ningún análisis, y solo a nivel informativo se presenta a continuación una tipología de los sistemas de educación a distancia, que existen en la actualidad. Esta esquematización se basa en algunos de los trabajos presentados en la conferencia, particularmente el documento No. 53 elaborado por Kaye and Rumble.

El primer modelo, ejemplificado desde hace más de cien años, en la tradición de Cambridge y Oxford en Inglaterra, consiste en la provisión de enseñanza por correspondencia ofrecida por una organización independiente y cuyo titulo es otorgado por una universidad pública. Este modelo aun existe en algunos países europeos.

Un segundo modelo es el que ofrecen algunas universidades convencionales, donde se brindan facilidades de estudio independiente, con acreditación y exámenes, a estudiantes regulares. En tal modalidad se pueden considerar universidades que promueven estudios por correspondencia en un solo departamento, como por ejemplo la Escuela de Educación en la Universidad del Pacifico Sur; universidades en las cuales los departamentos deben admitir tanto estudiantes presénciales (sistema convencional como estudiantes por correspondencia, pero con una responsabilidad aparte para asuntos administrativos; las universidades de New England en Australia y de Zambia son un modelo de esto y universidades que poseen unidades separadas de enseñanza por correspondencia con funciones administrativas y docentes, tales como la Universidad de Queenland en Canadá, de Panjab en la India y muchas universidades en los Estados Unidos, de las cuales Wisconsin constituye el ejemplo mejor conocido. 
El tercer modelo de educación superior a distancia es aquel en el cual colaboran varias instituciones en la atención de los estudiantes no presénciales. Una muestra de este tipo es la Universidad de Massey, en Nueva Zelandia, que ofrece educación a distancia a estudiantes de otras universidades del país; como también, los grupos regionales de las universidades francesas y el Instituto Alemán de Estudios a Distancia de Tubingen que ofrecen su sistema en colaboración con otras universidades y entidades de radiotelecomunicaciones.

El cuarto modelo está definido por una institución central que ofrece educación a distancia en todos los niveles de enseñanza. Tal es el caso del Centro Nacional de Teleeducación en Francia, bajo la dependencia del Ministerio de Educación, que cuenta con 200.000 estudiantes, de los cuales 5.000 persiguen un título académico.

Finalmente, el modelo que constituye quizá el desarrollo más reciente y el de mayor impacto, es aquel consistente en instituciones autónomas de educación superior, únicamente para estudiantes a distancia y que ofrece mediante una combinación especial de medios, diversos niveles de educación con responsabilidad formal para su evaluación y promoción. La primera de esta nueva generación de instituciones fue la Universidad Abierta de Inglaterra (The Open University), denominada así, por no tener requisitos de ingreso y por su apertura en cuanto a métodos de enseñanza. Otros ejemplos más recientes de esta modalidad son la Universidad Nacional de Educación a Distancia de España (UNED), la Universidad a Distancia de Alemania Occidental, la Universidad Libre de Irán, la Universidad para cada Hombre de Israel, la Universidad Abierta Allama Igbal de Pakistán, la Universidad Estatal a Distancia de Costa Rica (UNED), la Universidad Nacional Abierta de Venezuela (UNA) y la Universidad de Athabasca en Canadá.

Por la importancia y actualidad de esta modalidad, señalaremos brevemente algunos rasgos de este modelo de institución autónoma: a) las funciones de enseñanza, evaluación y certificación están integradas; b) la institución está totalmente comprometida con estudiantes no presénciales. De esta manera se evitan los conflictos de lealtades, en los sistemas convencionales y a distancia, entre el profesorado, y se facilita y acelera el desarrollo de métodos de enseñanza, al salirse del marco restringido de la enseñanza cara a cara; c) en principio, la institución es mucho más libre para diseñar y adaptar sus programas a las necesidades de grupos poblacionales, respondiendo mejor a éstas y optimizando su potencial educativo.

Como se señaló anteriormente, existe información disponible sobre los antecedentes y resultados de la conferencia, así como la colección de trabajos producidos con anterioridad a la reunión por parte de los participantes. Esta información está disponible para quienes así lo deseen. Cualquier solicitud se puede hacer al autor de esta breve noticia, a través de la dirección de la Revista.

Jairo Arboleda Toro 